\title{
ORIGINAL ARTICLE \\ Bladder management in individuals with chronic neurogenic lower urinary tract dysfunction
}

\author{
J Krebs ${ }^{1}$, J Wöllner ${ }^{2}$ and J Pannek ${ }^{2}$
}

Study Design: Retrospective investigation.

Objectives: To investigate the association of patient and injury characteristics with bladder evacuation by indwelling catheterization in patients with chronic neurogenic lower urinary tract dysfunction (NLUTD).

Setting: Tertiary urologic referral center.

Methods: The patient database was screened for patients with chronic ( $>12$ months) NLUTD. Patient characteristics and bladder management details were collected. Binary logistic regression analysis was used to investigate the effects of the investigated factors on bladder evacuation by indwelling catheterization.

Results: The data of 1263 patients with a median age of 47 years (range 11-89 years) and a median NLTUD duration of 15.2 years (range 1.0-63.4 years) were investigated. The most common bladder evacuation method was intermittent catheterization (IC; 41.3\%) followed by triggered reflex voiding $(25.7 \%)$, suprapubic catheterization (11.8\%), sacral anterior root stimulation (7.3\%), spontaneous voiding (7.0\%), abdominal straining (5.7\%) and transurethral catheterization (1.3\%). Female gender, tetraplegia, an age older than 45 years and injury duration were significant $(<0.001)$ predictors of indwelling catheterization. The odds of bladder evacuation by indwelling catheterization were increased $\sim 2.5,3$ and 4 times in women, patients older than 45 years and tetraplegics, respectively. Conclusions: IC is the most common bladder evacuation method. However, the majority of individuals with NLUTD are using other evacuation methods, because factors such as functional deficiencies, mental impairment or the social situation are relevant for choosing a bladder evacuation method. Individuals at risk of indwelling catheterization can be identified based on female gender, age, injury severity and injury duration.

Spinal Cord (2016) 54, 609-613; doi:10.1038/sc.2015.196; published online 10 November 2015

\section{INTRODUCTION}

The primary goal of bladder management in individuals with neurogenic lower urinary tract dysfunction (NLUTD) is to achieve low urine storage and voiding pressures, in order to protect renal function. ${ }^{1}$ The standard strategy to achieve this goal is bladder evacuation by intermittent catheterization (IC) and, in individuals with neurogenic detrusor overactivity (NDO), detrusor relaxation either by antimuscarinic (AM) treatment or by botulinum toxin (BTx) injections into the detrusor. ${ }^{1}$ If insufficient manual dexterity or other reasons preclude IC, triggered reflex voiding (RV) in men or suprapubic catheterization (SPC) in women may represent alternative evacuation methods. ${ }^{2-4}$ IC by a caregiver is only rarely accepted by individuals with NLUTD because of the loss of independence. ${ }^{5}$

Bladder evacuation by IC may be the most prevalent evacuation method at discharge from rehabilitation; ${ }^{2,6,7}$ however, some individuals seem to change their evacuation method over time. ${ }^{6,7}$ The reported rate of individuals who abandon IC ranges from 20 to $52 \%$, and most of them seem to revert to an indwelling catheter. ${ }^{6,7}$ Yet, indwelling catheters are associated with an increased risk of complications such as bladder cancer, ${ }^{8,9}$ bladder stones ${ }^{10-13}$ and urinary tract infections. ${ }^{14,15}$

We have therefore investigated the association of patient and injury characteristics with the bladder evacuation method in individuals with chronic NLUTD. The following hypotheses were tested: gender, age, injury severity and injury duration have a significant effect on bladder evacuation with an indwelling catheter.

\section{SUBJECTS AND METHODS}

\section{Subjects and collected data}

The present study had been approved by the local ethics committee, and all applicable institutional and governmental regulations concerning the ethical use of the data were followed.

The patient database of a tertiary urologic referral center was screened for patients with NLUTD who had presented for a routine urodynamic investigation between April 2008 and March 2012. Patients with a NLUTD duration shorter than 1 year or a NLUTD etiology other than traumatic or nontraumatic spinal cord injury (SCI) or myelomeningocele were excluded.

Patient characteristics, bladder diary details, the occurrence of NDO based on urodynamic investigation, the type of detrusor relaxation treatment and surgical interventions were collected from paper and electronic patient charts. If the bladder evacuation method had been changed less than 12 months ago (for example, transurethral indwelling catheterization (TUC) after a surgical intervention), the previous evacuation method was recorded for the analysis.

\section{Statistical analyses}

According to international recommendations, ${ }^{16}$ the investigated patients were grouped for age and injury duration using 15- and 5-year increments,

${ }^{1}$ Clinical Trial Unit, Swiss Paraplegic Centre, Nottwil, Switzerland and ${ }^{2}$ NeuroUrology, Swiss Paraplegic Centre, Nottwil, Switzerland Correspondence: Professor Dr J Pannek, NeuroUrology, Swiss Paraplegic Centre, Guido A. Zäch Strasse 1, Nottwil CH-6207, Switzerland. E-mail: juergen.pannek@paraplegie.ch

Received 4 August 2015; revised 18 September 2015; accepted 22 September 2015; published online 10 November 2015 
respectively. Furthermore, the injury severity was classified into four groups: (1) American Spinal Injury Association Impairment Scale (AIS) D, (2) AIS A-C paraplegia, (3) AIS A-C low-level tetraplegia (C8-C5) and (4) AIS A-C highlevel tetraplegia $(\mathrm{C} 4-\mathrm{C} 1)$. The effects of gender, age, injury severity and injury duration on bladder evacuation by SPC or TUC were investigated using binary logistic regression analysis. Fisher's exact test was used to investigate differences in the observed frequencies between groups (gender, age, injury severity, injury duration and etiology). The statistical analyses were performed using the SPSS software (version 18.0.3, IBM, Somers, NY, USA). A $P$-value of $\leqslant 0.05$ was considered significant.

\section{RESULTS}

A total of 1421 patients had presented at our department at least once between April 2008 and March 2012 for a routine urodynamic investigation. On the basis of the etiology of urinary tract dysfunction, 104 patients were excluded from the analysis (multiple sclerosis, $n=61$; cerebral palsy, $n=11$; Guillain-Barré syndrome, $n=10$; Friedreich's ataxia, $n=9$; other neurodegenerative disorders, $n=3$; locked-in syndrome, $n=4$; muscular dystrophy, $n=2$; peripheral neuropathy, $n=2$; stroke, $n=1$, and non-neurologic disorder, $n=1)$. Furthermore, 54 patients (45 men and 9 women) were excluded because the duration of NLUTD was shorter than 1 year (IC, $n=30$; SPC, $n=11$; spontaneous voiding, $n=5$; triggered RV, $n=4$, and abdominal straining, $n=4)$. Thus, the data of 1263 patients with a median age of 47 years (range 11-89 years) were investigated. The median duration of NLTUD was 15.2 years (range 1.0-63.4 years). A total of 951 (75.3\%) men and 312 (24.7\%) women were included. The etiology of NLUTD was mainly traumatic SCI (1085/88.9\%), followed by non-traumatic SCI (92/7.3\%) and myelomeningocele $(86 / 6.5 \%)$.

The most common bladder evacuation method was IC $(41.3 \%)$ followed by triggered RV (25.7\%), SPC (11.8\%), sacral anterior root stimulation $(7.3 \%)$, spontaneous voiding $(7.0 \%)$, abdominal straining
(5.7\%) and TUC (1.3\%; Table 1). No distinction was made between intermittent self and intermittent caregiver catheterization. Men mainly evacuated the bladder with IC (40.4\%) and triggered RV (31.4\%), whereas women predominantly used IC (43.9\%) and SPC (18.3\%; Table 1). In women, triggered RV was significantly $(P<0.0001)$ less common $(8 \%)$, but sacral anterior root stimulation $(12.8 \%)$ was significantly $(P<0.0002)$ more common compared with men (31.4 and 5.5\%). The odds of bladder evacuation by SPC or TUC in women were increased two and a half times compared with men (Table 2).

Triggered RV (33.7\%) and SPC (31.6\%) were the primary evacuation methods in high-level tetraplegics with AIS A-C (Table 1). In lowlevel tetraplegics with AIS A-C, IC was also quite common (19.9\%). More than half $(53.3 \%)$ of the paraplegics with AIS A-C evacuated their bladder with IC, and approximately one-fifth (19.2\%) used RV. The odds of bladder evacuation by SPC or TUC were increased approximately four and eight times in AIS A-C low- and high-level tetraplegics, respectively, compared with AIS D patients (Table 2).

The use of IC was significantly $(P \leqslant 0.009)$ decreased in patients older than 45 years, and bladder evacuation by SPC or TUC was significantly $(P=0.04)$ increased in patients older than 60 years (Table 3). The odds of bladder evacuation by SPC or TUC were increased approximately three times in patients older than 45 years (Table 2).

The injury duration had a negative effect on the use of IC resulting in significantly $(P<0.004)$ reduced proportions after 25 years of NLUTD (Figure 1). The proportion of bladder evacuation by RV was significantly $(P<0.02)$ increased after 5 years of NLUTD and stayed elevated thereafter at $\sim 35 \%$ (Figure 1 ). The use of SPC was significantly $(P<0.005)$ decreased after 5 years of NLUTD. After 30 years of NLUTD, the proportion of SPC increased gradually to the initial proportion, approximately (Figure 1).

Table 1 Distribution of bladder evacuation methods for gender and injury severity

\begin{tabular}{|c|c|c|c|c|c|c|c|c|}
\hline & $I C, \%(\mathrm{n})$ & $R V, \%$ (n) & $S P C, \%$ (n) & SARS, \% (n) & Spontaneous, \% (n) & Straining, \% (n) & TUC, \% (n) & Total, \% (n) \\
\hline \multicolumn{9}{|l|}{ Gender } \\
\hline Men & $40.4(384)$ & 31.4 (299) & $9.7(92)$ & $5.5(52)$ & $7.0(67)$ & $5.0(48)$ & $0.9(9)$ & $100(951)$ \\
\hline Women & 43.9 (137) & $8.0(25)^{*}$ & $18.3(57)^{*}$ & $12.8(40)^{*}$ & $7.1(22)$ & $7.7(24)$ & $2.2(7)$ & $100(312)$ \\
\hline \multicolumn{9}{|l|}{ Injury severity } \\
\hline C1-C4 AIS A-C & $17.3(17)$ & $33.7(33)^{* *}$ & $31.6(31)^{* * *}$ & $9.2(9)^{* * *}$ & $4.1(4)^{* * *}$ & $2.0(2)$ & $2.0(2)$ & $100(98)$ \\
\hline Men & $15.5(11)$ & $40.8(29)$ & $25.3(18)$ & $9.9(7)$ & $4.2(3)$ & $2.8(2)$ & $1.4(1)$ & $100(71)$ \\
\hline Women & $22.2(6)$ & $14.8(4)$ & $48.1(13)$ & $7.4(2)$ & $3.7(1)$ & $0.0(0)$ & $3.7(1)$ & $100(27)$ \\
\hline C5-C8 AIS A-C & $19.9(54)$ & $46.3(126)^{* * *}$ & $17.6(48)^{* * * *}$ & $11.0(30)^{* * *}$ & $2.9(8)^{* * *}$ & $0.7(2)^{* * *}$ & $1.4(4)$ & $100(272)$ \\
\hline Men & $18.3(39)$ & $57.7(123)$ & $13.6(29)$ & $8.5(18)$ & $0.9(2)$ & $0.5(1)$ & $0.5(1)$ & $100(213)$ \\
\hline Women & $25.4(15)$ & $5.1(3)^{*}$ & $32.2(19)^{*}$ & $20.3(12)^{*}$ & $10.2(6)^{*}$ & $1.7(1)$ & $5.1(3)^{*}$ & $100(59)$ \\
\hline T1-S5 AIS A-C & $53.3(385)^{* *}$ & 19.2 (139) & $8.4(59)$ & $7.3(53)^{* *}$ & $3.7(26)^{* * *}$ & $6.5(47)$ & $1.5(8)$ & $100(717)$ \\
\hline Men & $53.2(283)$ & 23.7 (126) & $7.5(38)$ & $5.1(27)$ & $3.8(20)$ & $5.8(31)$ & $0.9(5)$ & $100(530)$ \\
\hline Women & $53.4(102)$ & $6.8(13)^{\star}$ & $11.0(21)$ & $13.6(26)^{*}$ & $3.7(6)$ & $8.4(16)$ & $3.1(3)$ & 100 (187) \\
\hline AIS D & $31.1(41)$ & $15.9(21)$ & $8.3(11)$ & $0.0(0)$ & $34.8(46)$ & $8.3(11)$ & $1.5(2)$ & $100(132)$ \\
\hline Men & $32.7(36)$ & $16.4(18)$ & $6.4(7)$ & $0.0(0)$ & $34.5(38)$ & $8.2(9)$ & $1.8(2)$ & $100(110)$ \\
\hline Women & $22.7(5)$ & $13.6(3)$ & $18.2(4)$ & $0.0(0)$ & $36.4(8)$ & $9.1(2)$ & $0.0(0)$ & $100(22)$ \\
\hline NA & $54.5(24)$ & $11.4(5)$ & $0.0(0)$ & $0.0(0)$ & $11.4(5)$ & $22.7(10)$ & $0.0(0)$ & $100(44)$ \\
\hline Overall & $41.3(521)$ & 25.7 (324) & $11.8(149)$ & $7.3(92)$ & $7.0(89)$ & $5.7(72)$ & $1.3(16)$ & $100(1263)$ \\
\hline
\end{tabular}

Abbreviations: AIS, American Spinal Cord Injury Association Impairment Scale; C1-C4, cervical spinal cord segments 1 to 4. C5-C8, cervical spinal cord segments 5 to 8; IC, intermittent catheterization; NA, injury severity was not available for these patients; RV, triggered reflex voiding; SARS, sacral anterior root stimulation; SPC, suprapubic catheterization; Straining, voiding by abdominal straining (Valsalva); TUC, transurethral indwelling catheter; T1-S5, thoracic spinal cord segment 1 to sacral segment 5.

*Frequencies significantly $(P<0.04)$ different between genders are indicated in bold.

**Frequencies significantly different from the reference categories (AIS D, SCI) are indicated in bold: $P<0.015$

***Frequencies significantly different from the reference categories (AIS D, SCI) are indicated in bold: $P<0.0001$

**** Frequencies significantly different from the reference categories (AIS D, SCI) are indicated in bold: $P<0.035$. 
Interventions to reduce the bladder outlet resistance were performed in $24.3 \%$ of the patients (307/1,263): sphincterotomy $(n=243)$, bladder neck incision $(n=20)$ and urethral stent $(n=17)$. Interventions to improve urinary continence were performed in $5.4 \%$ of the patients $(68 / 1,263)$. The implantation of an artificial sphincter was the most common intervention $(n=61)$, followed by different sling techniques $(n=7)$.

The majority $(69.9 \%)$ of the patients (883/1263) suffered from NDO. In $54.9 \%$ of the patients with NDO (485/883), detrusor relaxation therapy was required. In all, 17 of the 485 patients (3.5\%) requiring detrusor relaxation-rejected treatment with $\mathrm{AM}$ or BTx. In 62.7\% (304/485) detrusor relaxation was achieved with AM,

Table 2 Binary logistic regression predicting bladder evacuation by suprapubic or transurethral catheterization from gender, age, injury severity and injury duration

\begin{tabular}{|c|c|c|c|}
\hline Predictors & P-value & $\operatorname{Exp}(B)$ & $95 \% \mathrm{Cl}, \operatorname{Exp}(B)$ \\
\hline Gender (men / women) & $<0.001$ & 2.55 & $1.74-3.74$ \\
\hline Age (years) ${ }^{a}$ & $<0.001$ & & \\
\hline $11-30$ & \multicolumn{3}{|c|}{ Reference category } \\
\hline $31-45$ & 0.19 & 1.60 & $0.79-3.22$ \\
\hline $46-60$ & 0.002 & 2.90 & $1.47-5.74$ \\
\hline $61-75$ & $<0.001$ & 7.76 & $3.82-15.76$ \\
\hline$>75$ & $<0.001$ & 10.45 & $4.10-26.61$ \\
\hline Injury severity ${ }^{a}$ & $<0.001$ & & \\
\hline C1-C4 AIS A-C & $<0.001$ & 8.27 & $3.76-18.16$ \\
\hline C5-C8 AIS A-C & $<0.001$ & 4.31 & $2.11-8.82$ \\
\hline T1-S5 AIS A-C & 0.24 & 1.51 & $0.77-2.96$ \\
\hline AIS D & \multicolumn{3}{|c|}{ Reference category } \\
\hline Injury duration (years) ${ }^{a}$ & 0.006 & & \\
\hline $1-5$ & \multicolumn{3}{|c|}{ Reference category } \\
\hline $6-10$ & 0.009 & 0.44 & $0.24-0.82$ \\
\hline $11-15$ & 0.002 & 0.37 & $0.20-0.69$ \\
\hline $16-20$ & 0.003 & 0.39 & $0.21-0.72$ \\
\hline $21-25$ & 0.012 & 0.39 & $0.19-0.82$ \\
\hline $26-30$ & 0.001 & 0.27 & $0.12-0.59$ \\
\hline $31-35$ & 0.038 & 0.44 & $0.20-0.95$ \\
\hline $36-40$ & 0.13 & 0.47 & $0.18-1.24$ \\
\hline $41-45$ & 0.08 & 0.41 & $0.16-1.10$ \\
\hline$>45$ & 0.06 & 0.38 & $0.14-1.02$ \\
\hline
\end{tabular}

Abbreviations: AIS, American Spinal Cord Injury Association Impairment Scale; $\mathrm{Cl}$, confidence interval; $\mathrm{C} 1-\mathrm{C} 4$, cervical spinal cord segments 1 to 4; C5-C8, cervical spinal cord segments 5 to $8 ; \operatorname{Exp}(\mathrm{B})$, odds ratio predicted by model; $\mathrm{T} 1-\mathrm{S} 5$, thoracic spinal cord segment 1 to sacral segment 5.

${ }^{a}$ General odds are not calculated for categorical predictors. $P$-values $\leqslant 0.05$ are indicated in bold. in $18.1 \%(88 / 485)$ with BTx injections into the detrusor and in $15.7 \%$ (76/485) with a combination of AM and BTx treatment. Ten patients underwent augmentation ileocystoplasty.

\section{DISCUSSION}

We have investigated the effects of patient and injury characteristics on bladder evacuation by an indwelling catheter in 1263 patients with chronic NLUTD (median duration 15.2 years). IC was the most common (41\%) bladder evacuation method followed by triggered RV $(26 \%)$ and SPC (12\%). Female gender, an age older than 45 years, AIS A-C tetraplegia and injury duration were significant predictors of bladder evacuation by SPC or TUC.

Intermittent self- or third-party catheterization is considered the standard bladder evacuation method for individuals with NLUTD, ${ }^{1}$ and the majority (that is, reported 46-74\%) of patients with NLUTD are discharged from primary rehabilitation using IC. ${ }^{6,7,17}$ However, the reported frequencies of IC following rehabilitation are very heterogeneous. In the present study, $41 \%$ of the patients used IC for bladder evacuation, which is in accordance with some other reports. ${ }^{6,17}$ Other authors have observed lower (that is, 15-33\%) 2,14,15,18 or higher (that is, 66-78\%) IC rates. ${ }^{19,20}$ The discrepancies are the result of differences in the study populations, study designs, national health-care systems and follow-up duration between the studies. The reported data concerning the rate of indwelling catheterization are even more diverse. Unfortunately, the comparison of study results is hampered, because some authors have not clarified whether 'indwelling catheterization' implies TUC and SPC or only TUC. In general, the reported historic rates of TUC are higher compared with the present results $(1.6 \%)$. Mainly, moderate rates (4-9\%) have been observed in European studies, ${ }^{2,19,20}$ whereas in other parts of the world reported TUC rates range from 17 to $41 \% .{ }^{14,15,18}$ The use of SPC seems to be similar $(11-14 \%)^{14,18}$ or less common $(4-9 \%)^{15,19}$ compared with present data $(13 \%)$.

After discharge from primary rehabilitation, some patients change their bladder evacuation method. Cameron et al. ${ }^{7}$ have observed a gradual decrease in the rate of IC from $46 \%$ at discharge to $14 \%$ 30 years after injury and an increase in indwelling catheterization (SPC and TUC) from 23 to $45 \%$. In the present study, the rate of IC had decreased from 48 to $19 \%$ after 45 years of NLUTD. The rate of SPC decreased after 5 years and then increased again after 30 years. The rate of TUC increased after 20 years of NLUTD. These results, however, should be considered cautiously, because they represent a cross-sectional rather than a longitudinal data set. However, the compliance with IC over time seems to be low (that is, 20-50\%).

Table 3 Utilized bladder evacuation methods for different age groups

\begin{tabular}{|c|c|c|c|c|c|c|c|c|}
\hline Age (years) & $I C, \%(n)$ & $R V, \%$ (n) & $S P C, \%(n)$ & SARS, \% (n) & Spontaneous, \% (n) & Straining, \% (n) & $T U C, \%$ (n) & Total, \% (n) \\
\hline $31-45$ & $47.0(175)$ & $28.8(107)$ & $7.3(27)$ & $7.8(29)^{*}$ & $4.0(15)$ & $3.8(14)$ & $1.3(5)$ & $100(372)$ \\
\hline $61-75$ & $31.3(70)^{* *}$ & $19.2(43)$ & $22.8(51)^{\star *}$ & 6.7 (15) & $10.7(24)^{* * *}$ & $8.0(18)$ & $1.3(3)$ & $100(224)$ \\
\hline$>75$ & $20.5(9)^{* * * *}$ & $22.7(10)$ & $25.0(11)^{* * * *}$ & $2.3(1)$ & $20.5(9)^{*}$ & $4.5(2)$ & $4.5(2)^{* * *}$ & $100(44)$ \\
\hline $11-89$ & $41.3(521)$ & 25.7 (324) & 11.8 (149) & $7.3(92)$ & $7.0(89)$ & $5.7(72)$ & $1.3(16)$ & 100 (1263) \\
\hline
\end{tabular}

Abbreviations: IC, intermittent catheterization; RV, triggered reflex voiding; SARS, sacral anterior root stimulation; SPC, suprapubic catheterization; Straining, voiding by abdominal straining (Valsalva); TUC, transurethral catheter.

Frequencies significantly different from the frequency of the reference category (11-30 years) are indicated in bold.

$* P \leqslant 0.003$.

$* * P \leqslant 0.0007$.

$* * * P \leqslant 0.04$.

$* * * * P \leqslant 0.005$ 


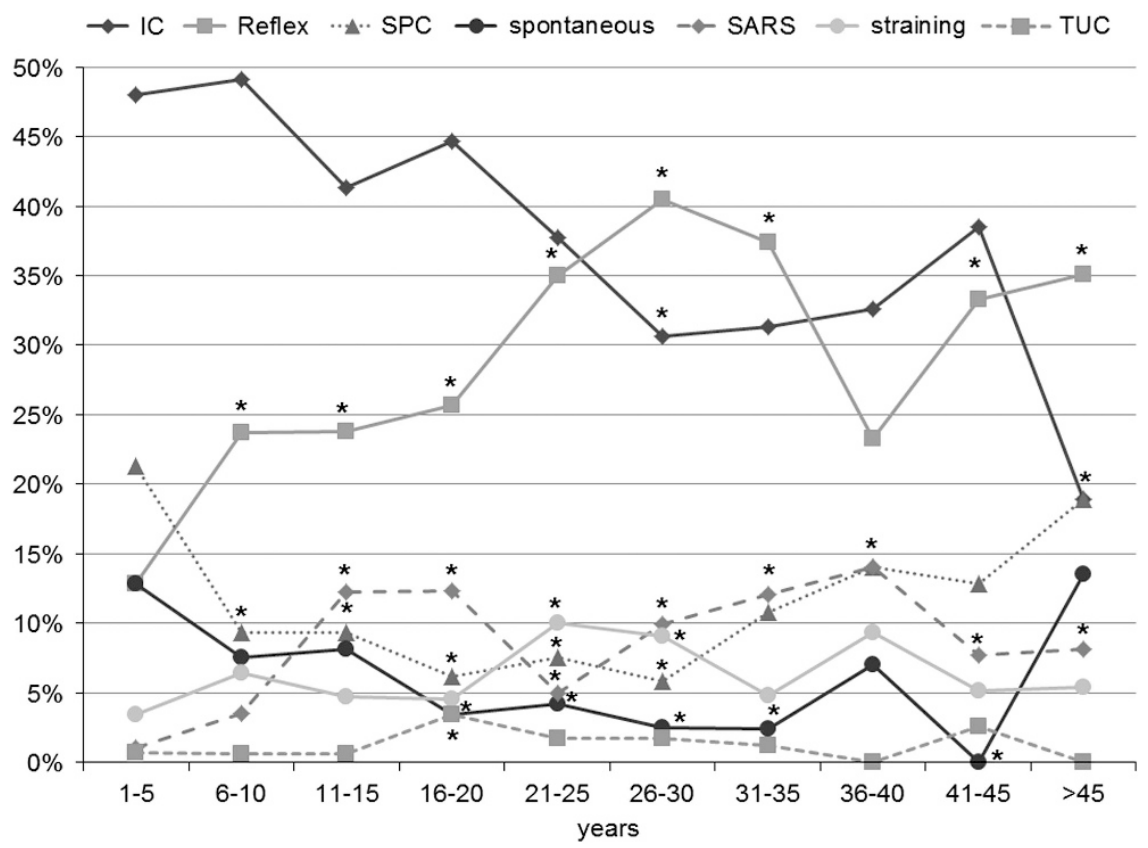

Figure 1 Frequencies of different bladder evacuation methods for different durations of neurogenic lower urinary tract dysfunction (NLUTD). IC, intermittent catheterization; Reflex, triggered reflex voiding; SARS, sacral anterior root stimulation; SPC, suprapubic catheterization; TUC, transurethral catheter. *Significantly $(P<0.04)$ different from the frequency for $1-5$ years duration of NLUTD.

The majority of individuals with NLUTD who abandon IC seem to change to indwelling catheterization. ${ }^{6,7}$ However, bladder evacuation by indwelling catheterization is associated with an increased risk of serious complications, ${ }^{8-10,12-15}$ and thus individuals at risk of changing to indwelling catheterization should be identified and alternative solutions should be offered.

In the present study, gender, age, injury severity and injury duration were significant predictors of bladder evacuation by SPC or TUC. In women, the odds of bladder evacuation by indwelling catheterization were increased two and a half times. This is in contrast to other authors who have not observed a significant gender effect on the type of bladder evacuation method or the compliance with IC after rehabilitation. ${ }^{6,20}$ However, evacuation by IC or RV is encumbered in women as a result of the anatomical situation. Thus, SPC is commonly (that is, $18 \%$ in present investigation) chosen as an alternative evacuation method. ${ }^{2-4}$

In AIS A-C tetraplegics, the odds of bladder evacuation by indwelling catheterization were increased up to eightfold. Evacuation by self-IC is possible in tetraplegics with a motor lesion level below C5 and sufficient wrist extensor muscle strength. ${ }^{21}$ In individuals with insufficient manual dexterity, third-party IC or RV (34\% in present investigation) may represent alternative methods. However, thirdparty IC entails logistic challenges and decreased independence, and urinary condom fixation problems may preclude RV in men and make it virtually impossible in women. Thus, indwelling catheterization is more commonly used in tetraplegics and in individuals with complete SCI. ${ }^{7,14}$ Consequently, IC compliance is lower in these individuals. ${ }^{6,14}$

In individuals older than 45 years, the odds of bladder evacuation by indwelling catheterization were increased approximately three times. The rate of SPC and TUC was significantly increased in individuals older than 60 (that is, 23\%) and 75 years (that is, $4.5 \%$ ), respectively. Cameron et al. ${ }^{7}$ have reported increased odds of indwelling catheterization (SPC and TUC) in patients older than 43 years at discharge from primary rehabilitation. Possible reasons for the increased use of indwelling catheters in elderly individuals are decreased dexterity, agility and cognitive capabilities, increased morbidity and dependence on caregivers. Furthermore, in elderly men, outlet obstruction caused by benign prostatic hypertrophy may preclude bladder evacuation by $\mathrm{RV}$ and force these individuals to resort to indwelling catheterization.

In the present study, $~ 70 \%$ of the patients suffered from NDO and $\sim 52 \%$ required detrusor overactivity relaxation therapy. These are similar rates compared with previously reported data (that is, $64 \%$ $\mathrm{NDO}$ or $63 \%$ requiring $\mathrm{AM}){ }^{6,20}$ In the majority of patients (that is, $65 \%$ ), sufficient detrusor relaxation was achieved with AM. In individuals with refractory NDO (that is, 31\%), BTx injections into the detrusor were successful in improving bladder capacity, storage and voiding pressures as well as overactivity symptoms. ${ }^{22}$ However, some individuals (that is, $16 \%$ ) required AM treatment additionally to the BTx injections in order to suppress NDO. Intolerable side effects of the AM were the main reason why $3.5 \%$ of the NDO individuals rejected detrusor relaxation treatment.

The causality between predictors and bladder evacuation by indwelling catheterization should be considered with caution because of the cross-sectional study design. Furthermore, some possible risk factors for a change to indwelling catheterization, such as for example the health-care system or the social situation, were not included in our analysis. Finally, the reported odds ratios should be interpreted with caution because of the wide confidence intervals.

On the basis of current literature, ${ }^{1}$ bladder evacuation by IC should be the standard method for individuals with voiding problems. However, in reality, the majority of individuals with NLUTD (that is, 59\%) are using evacuation methods other than IC. Anatomical obstacles, impaired manual dexterity, agility or cognitive capabilities, increased morbidity or dependence on caregivers are possible reasons why another evacuation method is chosen or implemented. In our cohort, gender, age and injury duration were identified as risk factors for indwelling catheterization. As the average age of individuals with 
NLUTD is increasing in line with an aging general population at risk, ${ }^{23,24}$ the occurrence of these risk factors will increase. In selected aging or multi-morbid individuals, SPC represents an appropriate alternative evacuation method, provided that close urological monitoring is maintained. ${ }^{2,3}$ Urinary diversion is a good alternative in otherwise healthy individuals who are not able to perform IC. However, in the patient group mentioned above, the general health condition usually precludes such surgical techniques. In selected cases, less invasive procedures, like a vesicostomy, may be able to prevent long-term indwelling catheterization. ${ }^{1}$ Bladder management is a holistic rehabilitation process, aiming at preserving both the renal function and quality of life. Thus, besides urodynamic findings, many other factors, such as functional deficiencies, physical or mental impairment or the social situation, are relevant for choosing a bladder evacuation method.

\section{CONCLUSIONS}

IC is the most common bladder evacuation method; however, the majority of individuals with NLUTD are using other evacuation methods. Individuals at risk of indwelling catheterization can be identified based on female gender, age, injury severity and injury duration.

\section{DATA ARCHIVING}

There were no data to deposit.

\section{CONFLICT OF INTEREST}

The authors declare no conflict of interest.

1 Blok B, Pannek J, Castro-Diaz D, Del Popolo G, Groen J, Gross T et al. Guidelines on Neuro-Urology. (European Association of Urology, Bern, Switzerland, March 2015).

2 El-Masri WS, Chong T, Kyriakider AE, Wang D. Long-term follow-up study of outcomes of bladder management in spinal cord injury patients under the care of the Midlands Centre for Spinal Injuries in Oswestry. Spinal Cord 2012; 50: 14-21.

3 Bothig R, Hirschfeld S, Thietje R. Quality of life and urological morbidity in tetraplegics with artificial ventilation managed with suprapubic or intermittent catheterisation. Spinal Cord 2012; 50: 247-251.

4 Thorup J, Biering-Sorensen F, Cortes D. Urological outcome after myelomeningocele: 20 years of follow-up. BJU Int 2011; 107: 994-999.
5 Vaidyanathan S, Soni BM, Singh G, Oo T, Hughes PL. Barriers to implementing intermittent catheterisation in spinal cord injury patients in Northwest Regional Spinal Injuries Centre, Southport, UK. Scientific WorldJournal 2011; 11: 77-85.

6 Yavuzer G, Gok H, Tuncer S, Soygur T, Arikan N, Arasil T. Compliance with bladder management in spinal cord injury patients. Spinal Cord 2000; 38: 762-765.

7 Cameron AP, Wallner LP, Tate DG, Sarma AV, Rodriguez GM, Clemens JQ. Bladder management after spinal cord injury in the United States 1972 to 2005. J Urol 2010; 184: 213-217.

8 West DA, Cummings JM, Longo WE, Virgo KS, Johnson FE, Parra RO. Role of chronic catheterization in the development of bladder cancer in patients with spinal cord injury. Urology 1999; 53: 292-297.

9 Stonehill WH, Dmochowski RR, Patterson AL, Cox CE. Risk factors for bladder tumors in spinal cord injury patients. J Urol 1996; 155: 1248-1250.

10 Bartel P, Krebs J, Wollner J, Gocking K, Pannek J. Bladder stones in patients with spinal cord injury: a long-term study. Spinal Cord 2014; 52: 295-297.

11 Mitsui T, Minami K, Furuno T, Morita H, Koyanagi T. Is suprapubic cystostomy an optimal urinary management in high quadriplegics?. A comparative study of suprapubic cystostomy and clean intermittent catheterization. Eur Urol 2000; 38: 434-438.

12 Ord J, Lunn D, Reynard J. Bladder management and risk of bladder stone formation in spinal cord injured patients. J Urol 2003; 170: 1734-1737.

13 Cameron AP, Wallner LP, Forchheimer MB, Clemens JQ, Dunn RL, Rodriguez G et al. Medical and psychosocial complications associated with method of bladder management after traumatic spinal cord injury. Arch Phys Med Rehabil 2011; 92: 449-456.

14 Rabadi MH, Aston C. Complications and urologic risks of neurogenic bladder in veterans with traumatic spinal cord injury. Spinal Cord 2015; 53: 200-203.

15 Singh R, Rohilla RK, Sangwan K, Siwach R, Magu NK, Sangwan SS. Bladder management methods and urological complications in spinal cord injury patients. Indian J Orthop 2011; 45: 141-147.

16 DeVivo MJ, Biering-Sorensen F, New P, Chen Y. Standardization of data analysis and reporting of results from the International Spinal Cord Injury Core Data Set. Spinal Cord 2011; 49: 596-599.

17 Afsar SI, Yemisci OU, Cosar SN, Cetin N. Compliance with clean intermittent catheterization in spinal cord injury patients: a long-term follow-up study. Spinal Cord 2013; 51: 645-649.

18 Weld KJ, Graney MJ, Dmochowski RR. Differences in bladder compliance with time and associations of bladder management with compliance in spinal cord injured patients. J Urol 2000; 163: 1228-1233.

19 Hagen EM, Rekand T. Management of bladder dysfunction and satisfaction of life after spinal cord injury in Norway. J Spinal Cord Med 2014; 37: 310-316.

20 Yildiz N, Akkoc Y, Erhan B, Gunduz B, Yilmaz B, Alaca R et al. Neurogenic bladder in patients with traumatic spinal cord injury: treatment and follow-up. Spinal Cord 2014; 52: 462-467.

21 Kriz J, Relichova Z. Intermittent self-catheterization in tetraplegic patients: a 6-year experience gained in the spinal cord unit in Prague. Spinal Cord 2014; 52: 163-166.

22 Ginsberg D, Gousse A, Keppenne V, Sievert KD, Thompson C, Lam W et al. Phase 3 efficacy and tolerability study of onabotulinumtoxinA for urinary incontinence from neurogenic detrusor overactivity. J Urol 2012; 187: 2131-2139.

23 Bellucci CH, de Castro Filho JE, Gomes CM, de Bessa J Jr, Battistella LR, Rubio de Souza $\mathrm{D}$ et al. Contemporary trends in the epidemiology of traumatic spinal cord injury: changes in age and etiology. Neuroepidemiology 2015; 44: 85-90.

24 Nijendijk JH, Post MW, van Asbeck FW. Epidemiology of traumatic spinal cord injuries in The Netherlands in 2010. Spinal Cord 2014; 52: 258-263. 